\title{
Enhanced CORDIC Based Rotator Design for Sinusoidal Transforms
}

\author{
Trivedi Pratik, Zaveri Tanish
}

\begin{abstract}
Transforms play an important role in conversion of information from one domain to the other. To be more specific transforms like Discrete Fourier transform (DFT) and Discrete Cosine transform (DCT) helps us to migrate from one time domain to frequency domain based on the basis function selected. The basis function of the every sinusoidal transform carries out a circular rotation to convert information from one domain to the other. There are applications related to communication which requires this rotation into the hyperbolic trajectory as well. Multiplierless algorithm like CORDIC improves the latency of the transforms by eliminating the number of multipliers in the basis function. In this paper we have designed and implemented enhanced version of CORDIC based Rotator design. The Enhanced version is simulated for order 1 to order 36 to emphasize on the results of the proposed algorithm. Results shows that the enhanced CORDIC rotator design surpasses the Mean square error after the order 18 compared to standard CORDIC. Unified CORDIC also can be implemented using the said algorithm to implement different three trajectories.
\end{abstract}

Keywords: CORDIC, DCT, DFT.

\section{INTRODUCTION}

Multipliers play an important role in digital signal processing applications now a days as people are hungrier about data and hence the amount of information that needs to be processed is very large and the time required to process that amount is limited. In such kind of cases it becomes critical to use multiplier based algorithms and hence CORDIC[1] and related multiplier less algorithms are popular since their inception. However CORDIC based approaches have certain drawbacks like the number of iterations and scaling factor. Unified CORDIC[7] approach takes this algorithm further by adding just a parameter and increasing the trajectory from only circular to linear, circular and hyperbolic.

The unified approach is very crucial if large number of applications needs to be covered by a single tunable hardware for all three different trajectories. The moment Unified CORDIC[7] combines the three approached the problem of

Revised Manuscript Received on February 05, 2020.

* Correspondence Author

Pratik Trivedi*, Electronics and Communication engineering department, Institute of Technology, Nirma University, Ahmedabad, India Email: erpratik84@gmail.com

Zaveri Tanish, Electronics and Communication engineering department, Institute of Technology, Nirma University, Ahmedabad, India Email: er_pratik84@yahoo.co.in

(C) The Authors. Published by Blue Eyes Intelligence Engineering and Sciences Publication (BEIESP). This is an open access article under the CC BY-NC-ND license (http://creativecommons.org/licenses/by-nc-nd/4.0/)

ROC(region of convergence) comes into picture as all the three trajectories ROCs are not matched to a single architecture. It is extremely important to optimise the number of hardware components used by Unified CORDIC[7] and also their ROCs needs to be matched in all cases. Here we propose an architecture which combines the three trajectories and takes care of the ROCs as well.

Section II. gives an overview on the basics of CORDIC[1] algorithm, section III talks about Unified CORDIC[1], section IV discuss on our approach and $\mathrm{V}$ focuses on results and analysis followed by conclusion and references.

\section{CORDIC ALGORITHM[1]}

CORDIC[1] stands for the Co-ordinate Rotation In Digital Computer. It tries to bring down the multipliers used in the rotators by shift and add approach. The equations (1) shows the normal rotation for points $x i$ and $y i$ by and angle $\theta \&$ equation (2) below show the normal operation of CORDIC[1] algorithm. As we can see that there are some assumptions taken into consideration in equation (1) to convert it into shift and add approach. It has an advantage of having only a single multiplication of scaling factor if the number of iterations are comparatively higher.

$$
\begin{gathered}
{\left[\begin{array}{l}
X i+1 \\
Y i+1
\end{array}\right]=\left[\begin{array}{l}
x i \\
y i
\end{array}\right]\left[\begin{array}{cc}
\cos \theta i & -\sin \theta i \\
\sin \theta i & \cos \theta i
\end{array}\right](1)} \\
{\left[\begin{array}{l}
X i+1 \\
Y i+1
\end{array}\right]=\left[\begin{array}{l}
x i \\
y i
\end{array}\right] \cos \theta i\left[\begin{array}{cc}
1 & -\tan \theta i \\
\tan \theta i & 1
\end{array}\right]}
\end{gathered}
$$

Where $\tan \theta i=2^{-i}$

\section{UNIFIED CORDIC[7]}

CORDIC algorithms can then be computed with help of linear, circular, and hyperbolic trajectories. The equations (3) \& (4) describes that by adding a parameter $m$ to the basic CORDIC, it can be made to operate in three different trajectories viz. circular, linear and hyperbolic.

$$
\begin{aligned}
& X i+1=x i-m d i y i 2^{-i} \\
& Y i+1=y i+d i x i 2^{-i}
\end{aligned}
$$

Where $m=0,1$ and -1 for linear circular and hyperbolic trajectories. Circular and hyperbolic find various applications and used extensively. CORDIC in circular trajectory is mainly used for the computation of sinusoidal functions, it also finds it applications in transform calculations, whereas, hyperbolic version of CORDIC is mainly applicable for finding the exponents and neural networks. 


\section{Enhanced CORDIC Based Rotator Design for Sinusoidal Transforms}

\section{ENHANCED SCALING FREE CORDIC[3]}

The sine and cosine components present in the normal CORDIC equations can also be represented in the form on Taylor series expansion and can be written as

$$
\begin{aligned}
& \sin x=x-\frac{x^{3}}{3 !}+\frac{x^{5}}{5 !}-\frac{x^{7}}{7 !}+\cdots \\
& \cos x=1-\frac{x^{2}}{2 !}+\frac{x^{4}}{4 !}-\frac{x^{6}}{6 !}+\cdots
\end{aligned}
$$

This brings down the enhanced CORDIC equations as

$$
\begin{aligned}
& x i+1=x i\left(1-\frac{x^{2}}{2 !}+\frac{x^{4}}{4 !}-\frac{x^{6}}{6 !}+\cdots\right)-y i\left(x-\frac{x^{3}}{3 !}+\frac{x^{5}}{5 !}-\frac{x^{7}}{7 !}+\cdots\right) \\
& Y i+1=y i\left(1-\frac{x^{2}}{2 !}+\frac{x^{4}}{4 !}-\frac{x^{6}}{6 !}+\cdots\right)+x i\left(x-\frac{x^{3}}{3 !}+\frac{x^{5}}{5 !}-\frac{x^{7}}{7 !}+\cdots\right)
\end{aligned}
$$

\section{RESULTS AND DISCUSSIONS}

In order to evaluate the performance of standard and enhanced CORDIC for better accuracy and feasibility of transforms, the coefficients from both the approaches were calculated and their mean square error were found with respect to the expected value of coefficients. The equations depict the formula used for the MSE.

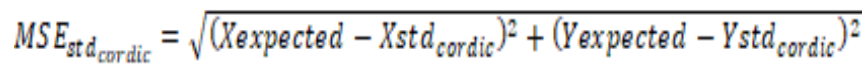

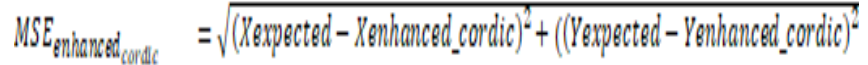

The MSE for 0-4096 points were calculated. Order $0,2,4,6,8,10,12,14,16$ and 18 were considered for calculating enhanced CORDIC.

\section{A. Results for Basic CORDIC[1] Algorithm}

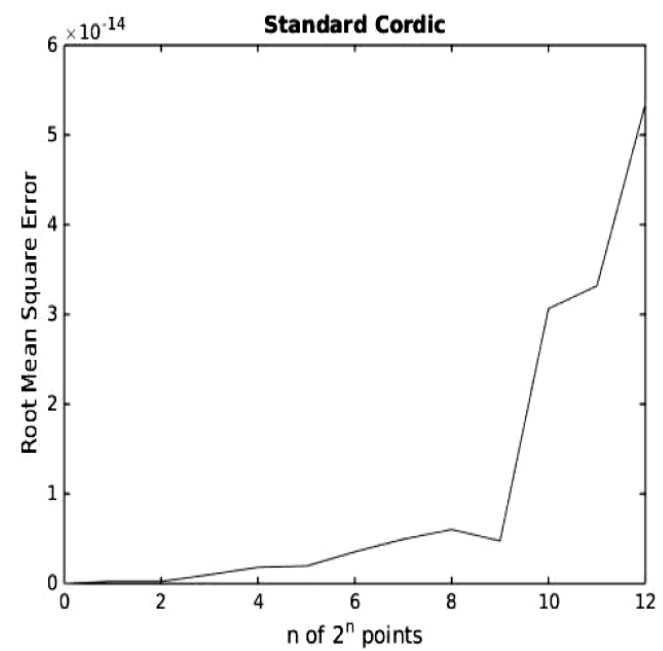

Fig.1.Mean Square error for N-point FFT for CORDIC Algorithm

From the above results, we could see that the MSE for standard CORDIC is in the order of $10^{-14}$ but the error goes on increasing as we increase the number of points for FFT.
This is only due to the error accumulation as we increase the number as the iterated number will be used to calculate the next one.

\section{B. Results for Enhanced CORDIC}

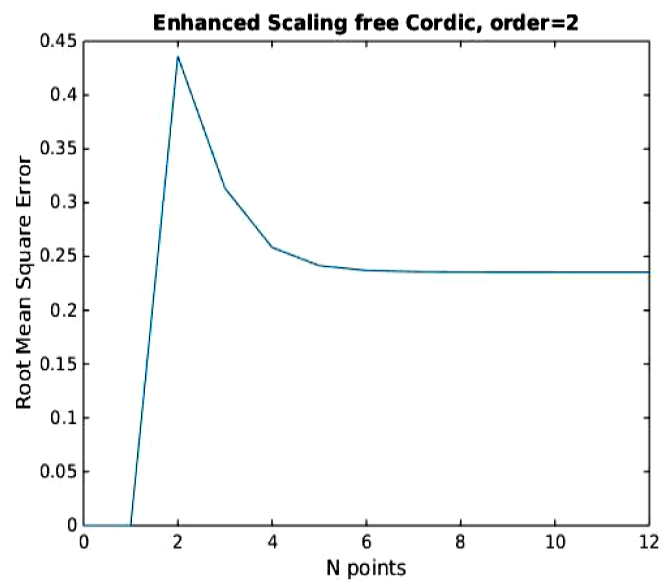

Fig. 2. Mean Square error for N-point FFT for Enhanced CORDIC Algorithm (2 ${ }^{\text {nd }}$ Order accuracy)

In Fig. 2 the error reduces with the number of points except that there is a spike at 2 point where we have already assumed the values to be 0 and 1 as the angle would be 0 and pi. But the error is very high in comparison with standard CORDIC[1] as it is in the order $10^{-2}$. 
Table-I Error Analysis for Standard CORDIC[1] and Enhanced CORDIC

\begin{tabular}{|c|c|c|c|c|c|c|c|c|c|}
\hline $\begin{array}{l}\text { Without } \\
\text { Shifter }\end{array}$ & WoSh & \multicolumn{8}{|c|}{ Error analysis for standard and Enhanced CORDIC with and without shifters } \\
\hline \multirow{2}{*}{$\begin{array}{l}\text { With } \\
\text { Shifters }\end{array}$} & WSh & & & & & & & & \\
\hline & \multicolumn{8}{|c|}{ Enhanced CORDIC } & $\begin{array}{l}\text { Standard } \\
\text { CORDIC[1] }\end{array}$ \\
\hline N Points & Order 2 & & Order 4 & & Order 6 & & Order 18 & & \\
\hline & WoSh & WSh & WoSh & WSh & WoSh & WSh & WoSh & WSh & \\
\hline 1 & 0 & 0 & 0 & 0 & 0 & 0 & 0 & 0 & 0 \\
\hline 2 & 0 & $\overline{0}$ & $\overline{0}$ & $\overline{0}$ & $\overline{0}$ & $\overline{0}$ & $\overline{0}$ & $\overline{0}$ & 2.5 e-16 \\
\hline 4 & 0.4361 & 0.4361 & 0.055 & 0.0683 & 0.0033 & 0.125 & $3.10 \mathrm{E}-14$ & 0.1211 & $2.30 \mathrm{E}-16$ \\
\hline 8 & 0.3135 & 0.3135 & 0.0389 & 0.05 & 0.0023 & 0.0895 & $2.19 \mathrm{E}-14$ & 0.0868 & $9.90 \mathrm{E}-16$ \\
\hline 16 & 0.2584 & 0.2584 & 0.0291 & 0.044 & 0.0017 & 0.0726 & $1.55 \mathrm{E}-14$ & 0.0707 & $1.81 \mathrm{E}-16$ \\
\hline 32 & 0.2416 & 0.2416 & 0.0251 & 0.0425 & 0.0013 & 0.0671 & $1.10 \mathrm{E}-14$ & 0.0656 & $1.95 \mathrm{E}-15$ \\
\hline 64 & 0.2371 & 0.2371 & 0.0239 & 0.0422 & 0.0012 & 0.0656 & $8.42 \mathrm{E}-15$ & 0.0642 & $3.54 \mathrm{E}-15$ \\
\hline 128 & 0.2359 & 0.2359 & 0.0236 & 0.0421 & 0.0012 & 0.0652 & $7.40 \mathrm{E}-15$ & 0.0639 & $4.94 \mathrm{E}-15$ \\
\hline 256 & 0.2356 & 0.2356 & 0.0236 & 0.0421 & 0.0012 & 0.0652 & 7.11E-15 & 0.0638 & $6.02 \mathrm{E}-15$ \\
\hline 512 & 0.2356 & 0.2356 & 0.0235 & 0.0421 & 0.0012 & 0.0651 & $7.03 \mathrm{E}-15$ & 0.0638 & 4.76E-15 \\
\hline 1024 & 0.2356 & 0.2356 & 0.0235 & 0.0421 & 0.0012 & 0.0651 & 7.01E-15 & 0.0638 & $3.06 \mathrm{E}-14$ \\
\hline 2048 & 0.2355 & 0.2355 & 0.0235 & 0.0421 & 0.0012 & 0.0651 & $7.00 \mathrm{E}-15$ & 0.0638 & $3.32 \mathrm{E}-14$ \\
\hline 4096 & 0.2355 & 0.2355 & 0.0235 & 0.0421 & 0.0012 & 0.0651 & $7.00 \mathrm{E}-15$ & 0.0638 & $5.35 \mathrm{E}-14$ \\
\hline
\end{tabular}

Table-I indicates the analysis of the Means square error calculated for standard CORDIC[1] and the proposed enhanced CORDIC starting from $\mathrm{N}=1$ till 4096 points and we can see till the $18^{\text {th }}$ order the error seems to be higher for enhanced CORDIC but it surpasses the Standard CORDIC after that as in case of standard CORDIC the error accumulates at each iteration.
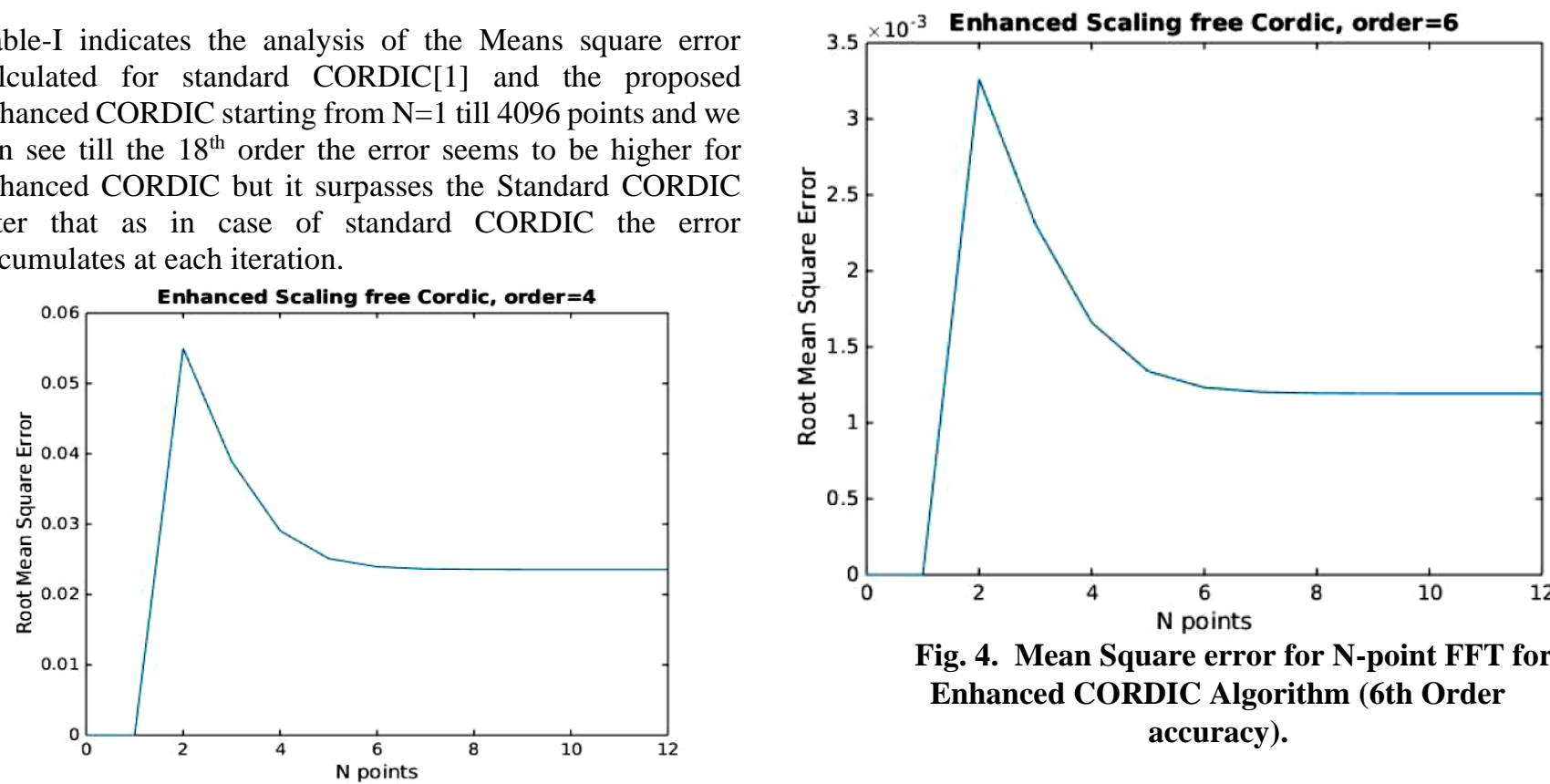

Fig. 4. Mean Square error for N-point FFT for Enhanced CORDIC Algorithm (6th Order accuracy).

Fig. 3. Mean Square error for N-point FFT for Enhanced CORDIC Algorithm (4th Order accuracy) 


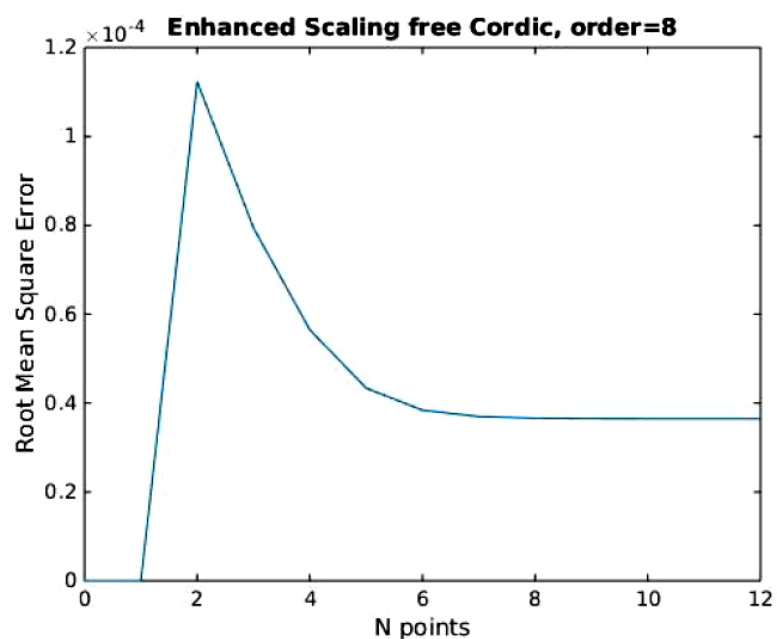

Fig. 5. Mean Square error for N-point FFT for Enhanced CORDIC Algorithm (8th Order accuracy)

Fig.3, 4 and 5 depicts the MSE for $4^{\text {th }}, 6^{\text {th }}$ and $8^{\text {th }}$ order enhanced CORDIC which indicates that the MSE reduces considerably to the order of $10^{-4}$ for the $8^{\text {th }}$ order. The figures shows us that using enhanced CORDIC the error goes on reducing as the number if points and the order goes on increasing.

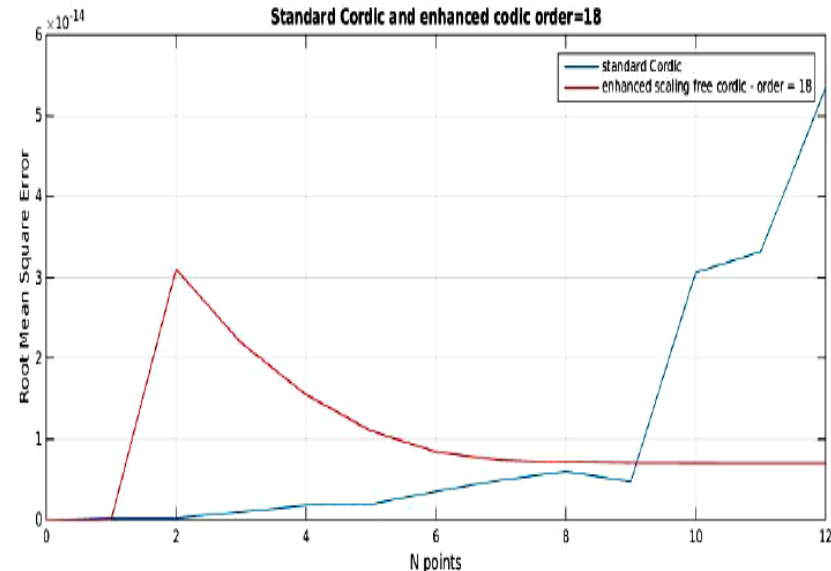

Fig. 5. Comparison for MSE for standard CORDIC and enhanced scaling free CORDIC

Fig. 5 clearly indicates that after the order 18 the enhanced scaling free CORDIC outperforms the standard CORDIC.

\section{CONCLUSIONS}

The results and discussions above shows that by increasing the order there is a considerable increase in accuracy compared to the basic CORDIC[1] algorithm. The Enhanced Scaling free CORDIC surpasses the basic CORDIC[1] at $18^{\text {th }}$ order of accuracy and also it is scaling free and hence one need not to multiply the constant at the end as well. This improves the latency of the transforms used in signal processing algorithms.

\section{REFERENCES}

1. J.E. Volder, "The CORDIC Trigonometric Computing Technique," IRE Transactions on Electronic Computers, vol. EC-8, no.3, pp. 330-334.

2. A G Dempster, M D Macleod, "Multiplication by two integers using the minimum number of adders," IEEE International symposium on circuits and systems,June 2005.

3. Francisco J. Jaime, Miguel A. Sánchez, Javier Hormigo, Julio Villalba, and Emilio L. Zapata, "Enhanced Scaling-Free CORDIC," IEEE TRANSACTIONS ON CIRCUITS AND SYSTEMS-I: REGULAR PAPERS, VOL. 57, NO. 7, JULY 2010.

4. Supriya Aggarwal, Pramod K. Meher, "Reconfigurable CORDIC Architectures for Multi-Mode and Multi-trajectory operations," IEEE International symposium on circuits and systems,June 2014.

5. Supriya Aggarwal , Pramod Kumar Meher, Senior Member, IEEE, and Kavita Khare, "Scale Free Hyperbolic CORDIC Processor and Its Application to Wave form Generation," IEEE TRANSACTIONS ON CIRCUITS AND SYSTEMS-I: REGULAR PAPERS, Vol. 60, NO. 2, February 2013.

6. Jens-Rainer Ohm, Gary J. Sullivan, Heiko Schwarz, Thiow Keng Tan, Thomas Wiegand, "Comparison of the Coding Efficiency of Video Coding Standards - Including High Efficiency Video Coding (HEVC) ,IEEE TRANS. ON CIRCUITS AND SYSTEMS FOR VIDEO TECHNOLOGY, Dec. 2012.

7. J.S. Walther, "A Unified Algorithm for Elementary Functions," Conference Proceedings, Spring Joint Computer Conference, May 1971, pp. 379-385.

\section{AUTHORS PROFILE}

Pratik Trivedi, is a Doctoral student at Institute of Technology, Nirma

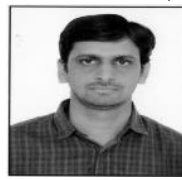

of ISTE.

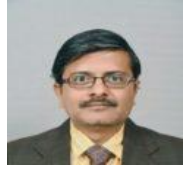

Dr. Tanish Zaveri, is a Professor at Institute of Technology, Nirma University. He completed his PhD from Sardar Vallabhbhai National Institute of Technology, Surat. He has published 25 papers in international conference and journals. His areas of interest are architectures for signal processing algorithms, Image processing, Machine learning etc. He is a member of IEEE. and applied sciences, Ahmedabad University. He has published 08 papers in various international conferences and journals. His areas of interest are architectures for signal processing Algorithms, Digital design etc. He is member of IEEE and lifetime member 